Ann. Zootech., I964, 13 (2), I65-I7I.

\title{
INFLUENCE DE L'INJECTION DE FER-DEXTRANE SUR LA COLORATION MUSCULAIRE DU JEUNE BOVIN
}

\author{
J. CHARPENTIER \\ avec la collaboration technique de Denise Gû́NE \\ Laboratoire de Recherches sur la Viande, \\ Centre national de Recherches zootechniques, Jouy-en-Josas (Seine-et-Oise)
}

\section{SOMMAIRE}

Une expérience portant sur 24 veaux de race Française Frisonne Pie Noire répartis en deux lots et soumis à une alimentation lactée exclusive, a été entreprise pour étudier l'effet d'injections intramusculaires de fer-dextrane sur la coloration musculaire. Les animaux ont été abattus à l'âge de trois mois. L'augmentation moyenne de la teneur en pigment musculaire des animaux traités par rapport a celle des témoins, varie, selon les muscles, de 60 à roo pour cent. Cette augmentation est relativement plus importante pour les animaux présentant une valeur élevée de l'hématocrite veineux à la naissance.

La production intensive de viande par de jeunes bovins abattus entre ro et I 8 mois pose actuellement de nombreux problèmes techniques et économiques. Les fréquentes difficultés de commercialisation de ces animaux sont imputables à la réaction défavorable de l'acheteur envers un type de viande qui ne présente pas les caractéristiques auxquels il est habitué.

L'insuffisance de coloration de ces viandes semble être le plus souvent, pour l'acheteur, le principal motif de disqualification.

Cette faible coloration est due essentiellement à la faible teneur en myoglobine qui caractérise d'une façon générale toutes les viandes jeunes.

Le groupement prosthétique de cette chromoprotéine, comme celui de l'hémoglobine du sang, présente la particularité de contenir du fer. Aussi nous a-t-il semblé judicieux de voir dans quelle mesure un apport supplémentaire de cet élément serait susceptible d'accroître la synthèse de myoglobine et par là même d'augmenter l'intensité de la coloration musculaire. L'incorporation du fer dans la myoglobine se fait à partir du fer fixé sur la sidérophiline plasmatique. L'injection de fer par voie veineuse ou intramusculaire permet un apport direct, mais les quantités en excès de celles 
qui peuvent se combiner à la sidérophiline ne sont pas fixées et sont rapidement éliminées. L'injection intramusculaire d'un complexe tel que le fer soit libéré très progressivement dans le circuit sanguin, permet de remédier à cet inconvénient. Aussi notre choix s'est-il porté sur une préparation qui présente ces propriétés : le complexe ferdextrane.

Pour que l'effet spécifique de cet apport de fer ne soit pas masqué par celui de l'apport alimentaire, nous avons appliqué ce traitement à des animaux soumis à une alimentation ferriprive constituée par un régime lacté exclusif.

\section{MATÉRIEL E'T MÉTHODES} 7 jours.

24 veaux de race Française Frisonne Pie Noire furent achetés à un âge compris entre 4 et

Comme, dans une étude antérieure, nous avons montré l'influence de la valeur de l'hématocrite à la naissance sur l'intensité de la pigmentation ultérieure du tissu musculaire, il convenait de connaître les hématocrites des animaux avant la constitution des lots. Le sang prélevé à la veine jugu laire était recueilli sur anticoagulant et centrifugé dans des tubes de WINTROBE à 4000 tours/minute pendant 30 minutes. Deux lots de i 2 animaux furent alors constitués de telle sorte que la variation de l'hématocrite soit voisine dans chaque lot. L'alimentation de tous les animaux fut exclusivement lactée. Les animaux du lot expérimental reçurent tous les dix jours une injection intramusculaire de ro $\mathrm{ml}$ d'une solution de fer-dextrane a $5 \mathrm{p}$. 100 (soit $500 \mathrm{mg}$ de fer). L'hématocrite veineux fut déterminé chaque semaine et immédiatement avant l'abattage. Les animaux furent abattus à un poids compris entre I Io et I $20 \mathrm{~kg}$. Les échantillons des muscles Adductor, Sartorius et Rectus Abdominis furent prélevés 24 heures après l'abattage. Le pourcentage de rémission à 540 m $\mu$ fut déterminé avec un réflectomètre Électrosynthèse SP 3 sur une coupe fraîche de ces muscles effectuée dans le sens des fibres. Le pigment musculaire total (myoglobine + hémoglobine résiduelle) fut dosé par la méthode d'HoRNSEY sous forme de chlorydrate d'hématine.

\section{RÉSULTATS - DISCUSSION}

\section{Io) Évolution de l'hémotocrite veineux}

- Chez tous les animaux témoins, 1'hématocrite diminue rapidement les deux premières semaines, puis plus lentement.

- Les animaux ayant reçu du fer présentent une évolution toute différente. L'hématocrite continue en effet à diminuer pendant la semaine suivant la première injection, puis se stabilise et croît ensuite de façon régulière. L'augmentation de l'hématocrite semble alors plus rapide chez les animaux présentant des valeurs initiales faibles (fig. I).

Comme le montre le tableau I, les valeurs moyennes des hématocrites avant 1'abattage sont significativement différentes dans les deux lots :

\section{$\left.2^{\circ}\right)$ Hématocrite et pigmentation musculaire}

Lors d'une étude antérieure nous avions mis en évidence l'existence d'une liaison entre l'hématocrite à l'abattage et la teneur en pigment musculaire.

Cette liaison se trouve confirmée dans la présente expérience (tab1. 2), ce qui 
TABLEAU I

Comparaison des valeurs moyennes de l'hémalocrite dans les 2 lots

\begin{tabular}{c|c}
\hline Sans fer dextrane & Avec fer dextrane \\
\hline$x=23,2$ & $x=38,4$ \\
$\mathrm{P} 1 \%=2,79$ & $t=6,46$ \\
\hline
\end{tabular}

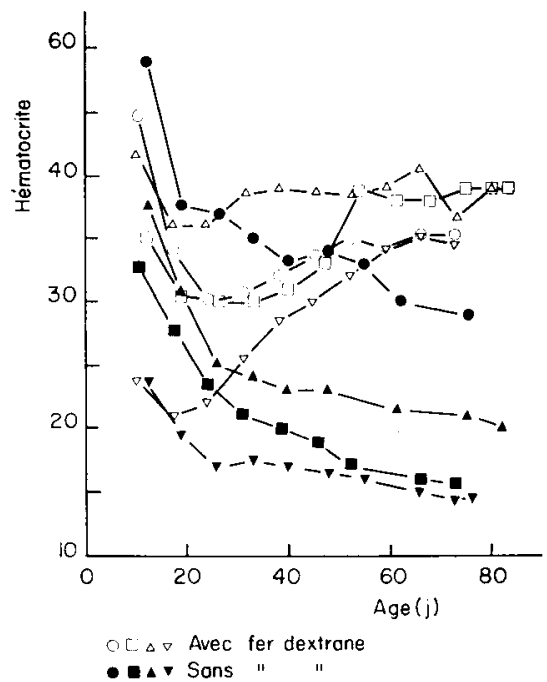

FIG. I. - Exemples d'évolution de l'hématocrite veineux chez quelques animaux

TABLEAU 2

Valeurs des coefficients de corrélation entre l'hématocrite à'l'abattage et la teneur en pigment de trois muscles

\begin{tabular}{|c|c|}
\hline Muscle & $\begin{array}{l}\text { Coefficient de corrélation : } \\
\text { hématocrite-pigment }\end{array}$ \\
\hline Adductor $\ldots \ldots \ldots \ldots \ldots$ & $+0,88$ \\
\hline Sartorius $\ldots \ldots \ldots \ldots \ldots$ & +0.75 \\
\hline Rectus Abdominis . . . . . . & $+0,83$ \\
\hline $\mathbf{P}=0,01$ & $r=0,496$ \\
\hline
\end{tabular}


laisse penser que la synthèse de l'hémoglobine circulante consécutive à l'apport de fer s'est accompagnée également d'une synthèse de myoglobine dans le tissu musculaire.

\section{$3^{\circ}$ Différence de coloration musculaire}

entre les animaux témoins et les animaux supplémentés en fer

Comme l'indique le tableau 3 , les teneurs en pigment musculaire diffèrent significativement dans les deux lots.

TABIEAU 3

Comparaison des valeurs moyennes des teneurs en pigment musculaire dans les deux lots

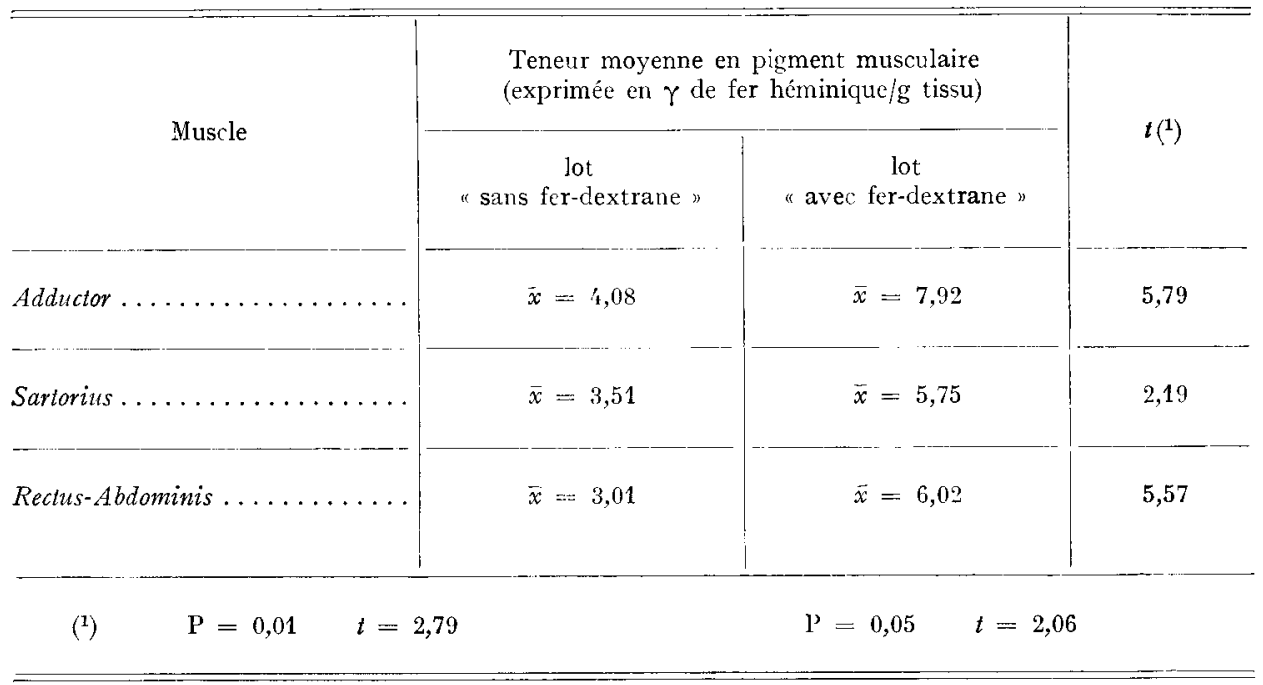

Les valeurs du pourcentage de rémission à $540 \mathrm{~m} \mu$ (qui traduisent objectivement l'impression de " clarté " de la surface de coupe) sont également très significativement différentes, comme le montre le tableau 4.

Ces différents résultats montrent que les injections répétées de fer-dextrane ont pour effet d'accroître très nettement la coloration musculaire.

L'influence de la valeur initiale de l'hématocrite se manifeste à la fois chez les animaux témoins, ce qui confirme les résultats antérieurs et chez les animaux supplémentés en fer (fig. 2). Il semble d'ailleurs que l'augmentation de la teneur en pigment soit relativement plus importante pour les animaux dont l'hématocrite à la naissance est élevé. Une expérience complémentaire avec un nombre supérieur d'animaux serait nécessaire pour confirmer ou non cette hypothèse.

$\left.4^{\circ}\right)$ Importance de l'augmentation de la teneur en pigment musculaire consécutive à l'injection de fer

$L_{\text {, }}$ augmentation relative de la teneur en pigment varie selon les muscles, comme le montrent le tableau 5, d'une part, et la figure 3 , d'autre part. 


\section{TABLEAU 4}

Comparaison des valeurs moyennes du pourcentage de rémission de la surface de coupe d'un muscle, dans les deux lots

\begin{tabular}{|c|c|c|c|}
\hline \multirow{2}{*}{ Muscle } & \multicolumn{2}{|c|}{ Valeur moyenne du \% de rémission } & \multirow{2}{*}{$t\left({ }^{(1)}\right.$} \\
\hline & $\begin{array}{c}\text { lot } \\
\text { "sans fer-dextrane }\end{array}$ & $\begin{array}{l}\text { lot } \\
\text { "avec fer-dextrane" }\end{array}$ & \\
\hline Adductor..$\ldots \ldots \ldots \ldots \ldots \ldots$ & $\bar{x}=35,0$ & $\bar{x}=23,2$ & 4,91 \\
\hline Sartorius $\ldots \ldots \ldots \ldots \ldots \ldots$ & $x=38,9$ & $\vec{x}=27,2$ & $2 / t, 6$ \\
\hline Rectus*Abdominis . . & $\bar{x}=39,5$ & $\bar{x}=28,6$ & 12,2 \\
\hline & $\mathrm{P}=0,01$ & $\mathrm{P}=0,05$ & \\
\hline
\end{tabular}

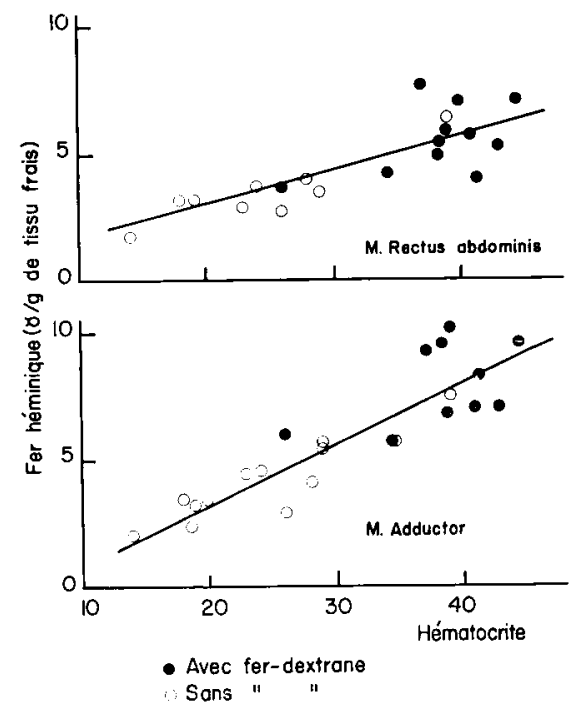

Fig. 2. - Liaison entre l'hématocrite initial et la teneur en pigment du muscle adducteur de la cuisse 
Le nombre insuffisant de données sur la pigmentation musculaire d'animaux plus âgés de la race utilisée dans cette étude ne nous permet pas d'apprécier comparativement avec exactitude l'augmentation de couleur consécutive à ce traitement.

Il semblerait que, dans les conditions de notre expérience, la supplémentation ferrique se traduise par un gain de coloration de l'ordre de quatre mois.

TABLFAU 5

Importance de l'augmentation de la teneur en pigment
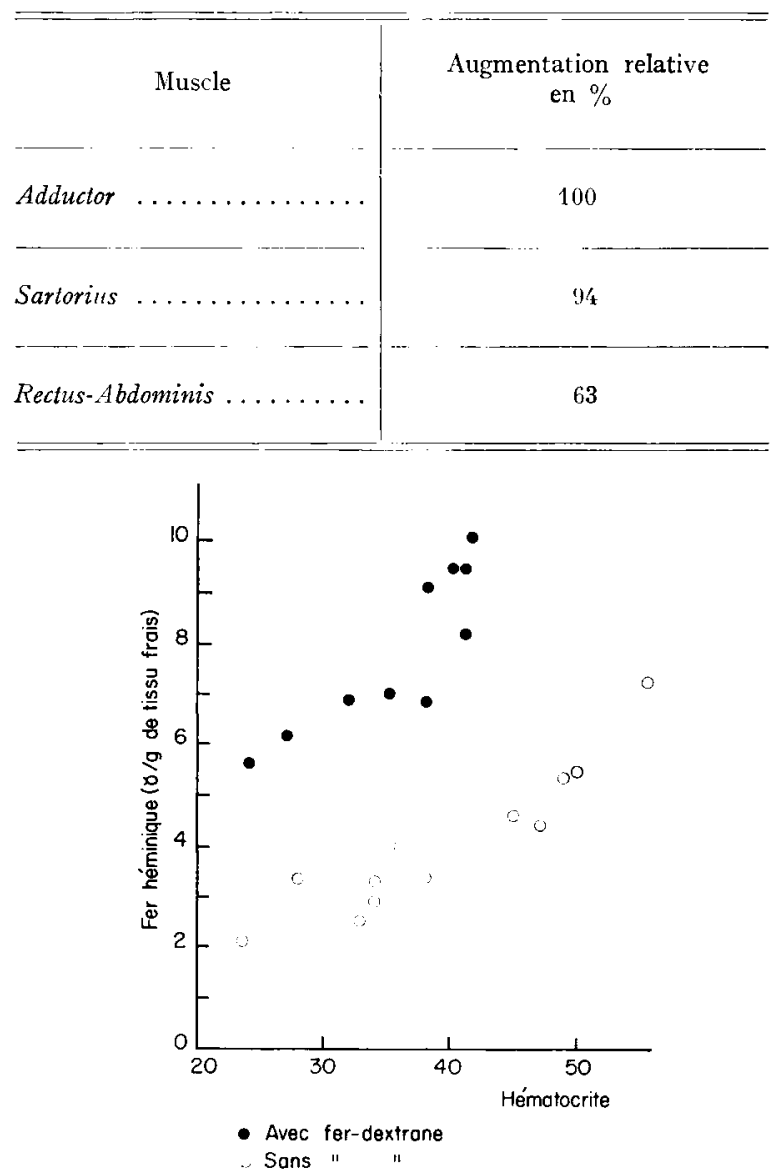

FIG. 3. - Liaison entre l'hématocrite d̀ l'abattage et la teneur en fer héminique musculaire

\section{CONCLUSION}

Les injections répétées de fer-dextrane provoquent chez le jeune bovin une nette augmentation de la teneur en pigment des muscles. L'influence de la valeur initiale de l'hématocrite sur la pigmentation musculaire, déjà mise en évidence dans 
une expérience antérieure, se manifeste encore dans le cas d'une importante supplémentation en fer.

Il semble donc que l'injection de fer dextrane puisse entraîner une amélioration de la couleur de la viande produite par de jeunes bovins soumis à une alimentation intensive. Les modalités techniques de cette supplémentation ferrique (fréquence des injections et doses) devront être précisées afin que cette méthode soit susceptible, dans l'avenir, d'une application pratique.

Il convient également de souligner que, par ailleurs, ce produit ne semble pas avoir d'effets nocifs, bien au contraire, puisqu'il est fréquemment utilisé en médecine vétérinaire pour combattre les états préanémiques, fréquents au cours des maladies infectieuses.

$$
\text { Rę̧u pour publication en janvier } 1964 \text {. }
$$

\section{SUMMARY \\ EFFECT OF THE INJECTION OF IRON DEXTRAN ON THE MEAT COLOUR OF THE CALF}

$24 F . F . P . N$. calves were alloted in two groups at one week of age and kept on a whole milk diet. All animals were slaughtered at three months. Iron dextran was injected intramuscularly at the rate of $500 \mathrm{mg}$ iron each tenth day to one of the lot. The colour of the meat was appreciated by the heminic iron level and the percent of reflectance for the Adductor, Sartorius and Rectus Abdominis muscles. The meat of the treated animals was much more coloured and the difference was highly significant. The heminic iron level in the treated animals was highly significantly increased. In spite of this iron supplementation, th influence of the hematocrit value at birth remained noticeable. The increase of the colour of meat seems to be greater in the animals which have a high hematocrit value at birth.

\section{RÉFÉRENCE BIBLIOGRAPHIQUE}

Hornsey, H. C., 1956. The colour of cooked cured pork. J. Sci. Food Agric., 7, 534-540. 